\title{
Chronic pain after SCI. A patient survey
}

\author{
A Ravenscroft*, ${ }^{*}$, YS Ahmed ${ }^{1}$ and IG Burnside ${ }^{1}$ \\ ${ }^{1}$ Department of Spinal Injuries, Pinderfields General Hospital, Wakefield, Yorkshire, UK
}

Study design: A survey of chronic pain experience after spinal cord injury.

Objective: To investigate the prevalence, severity and impact of chronic pain amongst spinal cord injury (SCI) patients in our region, and assess the need for additional resources to address the problem.

Methods: A postal questionnaire was sent to 216 spinal cord injury patients $(10 \%$ of the Yorkshire regional spinal injury database).

Setting: Yorkshire region, UK.

Results: A response was received from $67 \%$ of the patients. Seventy-nine per cent of patients said they presently suffered with pain, with $39 \%$ describing it as severe. Comparison of pain and non-pain groups using chi-squared analysis showed that complete injury was significantly more likely than incomplete injury to result in chronic pain $(P<0.05)$, and increased severity of pain $(P<0.05) .43 \%$ of patients with pain said they required further treatment for it. Chronic pain had a significant impact on daily activities and was a major factor in causing unemployment (18\%) and depression (39\%).

Conclusion: The study confirms that pain is a major problem in SCI patients which is not currently being adequately addressed. A multidisciplinary approach to management and prospective studies of treatments are required in order to reduce the prevalence and severity of pain in these patients.

Spinal Cord (2000) 38, 611-614

Keywords: pain; spinal cord injury; dyaesthesia; central pain

\section{Introduction}

Spinal cord injury effects approximately 900-1000 patients per million population. ${ }^{1,2}$ There is widespread appreciation of the physical disability that accompanies injury to the spinal cord. However, it is less well recognised that a significant proportion of these patients suffer with chronic pain.

Review of the current literature reveals inconsistency regarding the prevalence, aetiology and classification of chronic pain after spinal cord injury, and little guidance on its effective management. This uncertainty extends to those clinicians responsible for the management of spinal cord injuries. ${ }^{3}$

The present study aimed to investigate the prevalence, severity and impact of chronic pain amongst spinal injury patients in West Yorkshire, and assess the need for additional resources to address the problem.

\section{Methods}

A postal questionnaire was sent to 216 spinal cord injury patients. These were randomly chosen by selecting every tenth name on the Yorkshire regional

*Correspondence: A Ravenscroft, City Hospital, Hucknall Road, Nottingham, NG5 1PB, UK spinal injury database. The questionnaire consisted of 37 questions relating to general demographics, spinal injury, pain and treatments tried. For each question respondents were given a choice of several different answers identified by a tick box, but were able to make further comments in spaces provided. Two mailings were sent 3 months apart and altogether 6 months were allowed for data collection.

\section{Results}

One hundred and forty-six out of $216(67 \%)$ responses were received in total, 89 responses to the first mailing and an additional 56 to the second. One hundred and nine out of $146(75 \%)$ of the respondents were male and 37 out of $146(25 \%)$ were female. The time since injury ranged from 2 months to 38 years (mean 8.35 years) amongst patients (Figure 1).

The cause of injury was trauma related in 126 out of $146(86 \%)$ of patients, with falls 46 out of $146(31 \%)$ and road traffic accidents 44 out of $146(30 \%)$ the most common causes. Non traumatic injury occurred in 20 out of $146(14 \%)$ of cases. These included infection in seven patients, the result of a medical procedure in six, vascular occlusion in two and tumour in two 47 out of $146(32 \%)$ patients had undergone an 
operative procedure on the spine; the majority of these were performed around the time of injury.

The vertebral level at which spinal injuries occurred are shown in Table 1 Injury was classified as complete in 58 out of $146(40 \%)$ respondents (total loss of sensation and function below the injury level). Fiftynine out of $146(40 \%)$ injuries were classed as incomplete. In 29 out of $146(20 \%)$ this information was missing.

\section{Pain}

A total of 115 out of $146(79 \%)$ patients said they presently suffered with pain. All patients described pain that had started after their spinal injury, and had persisted for at least 4 months. Comparison of pain and non-pain groups was performed using chi-squared analysis. This showed that complete injury was significantly more likely than incomplete injury to result in chronic pain $(P<0.05)$, and increased severity of pain $(P<0.05)$. The presence of pain did not correlate with differences in age, sex, cause of injury or level of injury. There was no significant difference between the complete and incomplete injury groups with regard to sex, age or level of injury.

Of those patients with pain, Fifty seven out of 115 $(50 \%)$ said it constituted their worst medical problem, compared with 40 out of $115(35 \%)$ that had most difficulty coping with paralysis.

Eighty out of $115(69 \%)$ patients experienced two or more different types of pain. Regarding their worst type of pain, 73 out of $115(63 \%)$ said pain had begun within the first 6 months post injury, and a further 17 out of $115(15 \%)$ between 6 and 12 months (Table 2).
Nineteen out of $115(16 \%)$ patients said that their pain had improved spontaneously with time.

Pain was sensed above the level of the spinal injury in 16 out of $115(14 \%)$ patients, at the level of injury in 42 out of $115(37 \%)$ and below the level in 87 out of $115(76 \%)$.

On a 6 point verbal rating scale, 45 out of 115 $(39 \%)$ assessed their pain as severe, 41 out of 115 $(36 \%)$ as moderate and 15 out of $115(13 \%)$ as mild. Fourteen patients failed to answer this question. Twelve out of $115(10 \%)$ described suffering severe pain for more than 10 years.

Forty-five out of $115(39 \%)$ said that their pain was continuous throughout the day, while others suffered attacks lasting hours $(28 \%)$, minutes $(13 \%)$ or seconds $(6 \%)$. The most common terms used to describe pain amongst patients are summarised in Table 3.

Respondents identified exacerbating factors for their pain including muscle spasms (25\%), Activity (35\%), touching the painful area (17\%), 'getting worked up' and cold weather $(6 \%)$. The most common alleviating factors were massage $(27 \%)$, the application of heat $(21 \%)$, and drugs $(22 \%)$. One hundred out of 115 $(87 \%)$ patients had sought treatment for their pain (Table 4). Fifty out of $115(43 \%)$ patients said they wanted further treatment for their pain. Sixty-nine out of $146(47 \%)$ patients were unemployed or retired. Twenty seven out of $146(18 \%)$ cited pain, 62 out of $146(42 \%)$ paralysis, and 15 out of $146(10 \%)$ a combination of both as the main reason for their continuing unemployment. Pain interfered with a range of daily activities (Table 5).

Forty-five out of $115(39 \%)$ patients said that pain had caused them to become depressed.

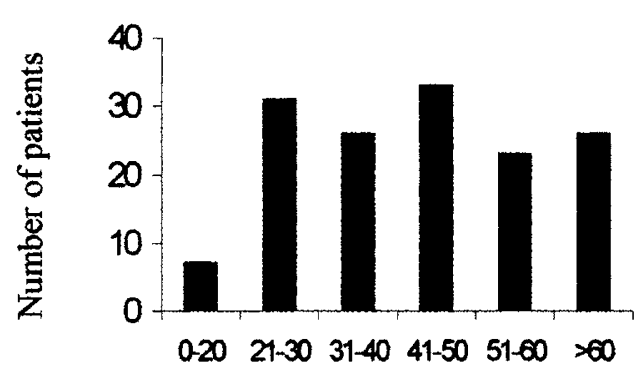

Age graup in years

Figure 1 Age distribution of patients

Table 1 Level of spinal cord injury

\begin{tabular}{lc}
\hline Level of injury & Patients (\%) \\
\hline Cervical (C1-C6) & $49(34)$ \\
Upper thoracic (T1-T6) & $29(20)$ \\
Lower thoracic (T7-L2) & $12(8)$ \\
Lumbar (below L2) & $25(17)$ \\
No data & $31(21)$ \\
\hline
\end{tabular}

Table 2 The amount of time patients had experienced pain

\begin{tabular}{lc}
\hline Duration of pain & Patients $(\%)$ \\
\hline 4 months -1 year & $20(17)$ \\
$1-3$ years & $30(26)$ \\
$3-10$ years & $40(35)$ \\
$>10$ years & $21(18)$ \\
No data & $4(3)$ \\
\hline
\end{tabular}

Table 3 The most common descriptors used by patients to describe their pain after SCI

\begin{tabular}{lc}
\hline Descriptor & Patients (\%) \\
\hline Aching & 60 \\
Hurting & 41 \\
Sore & 36 \\
Burning & 34 \\
Tight & 31 \\
Sharp & 29 \\
Tender & 28 \\
Stabbing & 28
\end{tabular}


Table 4 Treatments tried by patients in the past, and those that are still being used

\begin{tabular}{lcc}
\hline Treatments & $\begin{array}{c}\text { Tried } \\
(\%)\end{array}$ & $\begin{array}{c}\text { Still using } \\
(\%)\end{array}$ \\
\hline Paracetamol & 51 & 20 \\
Non steroidal anti-inflammatories & 30 & 7 \\
Weak opiates (eg coproxamol) & 57 & 23 \\
Baclofen & 26 & 12 \\
Antidepressants & 20 & 11 \\
Anticonvulsants & 14 & 7 \\
Opiates (eg morphine sulphate) & 14 & 1 \\
Transcutaneous nerve stimulation & 21 & 2 \\
Physiotherapy & 30 & 10 \\
Psychotherapy & 3 & 1 \\
Aromatherapy & 17 & 13 \\
Alternative therapy & 13 & 3 \\
Acupuncture & 8 & 1 \\
Cannabis & 5 & 5 \\
\hline
\end{tabular}

Table 5 Activities affected by pain

\begin{tabular}{lc}
\hline Pain interferes with & Patients $(\%)$ \\
\hline Sleep & 56 \\
Recreation/social life & 40 \\
Relationships & 14 \\
General activity & 28 \\
Therapy/rehabilitation & 13 \\
\hline
\end{tabular}

\section{Discussion}

Our study has shown a prevalence of chronic pain after SCI of $79 \%$, with $39 \%$ of these patients describing the pain as severe. Comparison of patient demographics with those of previous studies, ${ }^{1,2}$ suggest that our sample is representative of the SCI population.

Previous studies have estimated the prevalence of pain after SCI to range from $46 \%$ to $90 \%,{ }^{4-13}$ with $25 \%$ to $43 \%$ having severe pain. ${ }^{5-7,14}$ The reasons for this diversity are unclear, but may reflect inconsistencies in pain definition and measurement, patient reporting and consultant attitudes, as well as a genuine variation in prevalence. In addition, study samples may misrepresent the general SCI population by having small patient numbers ${ }^{12-14}$ and low response rates to questionnaires. ${ }^{5,8,10}$ Prevalence figures for chronic pain may also vary if estimates include patients with acute pain, ${ }^{13}$ 'abnormal sensations' and 'dyaesthesia, ${ }^{7}$ and those patients that have had pain since their SCI but do not have it now. ${ }^{4,6,11}$ We have addressed these issues by surveying a large number of patients, achieving a response rate of $67 \%$, and only including patients with ongoing pain of at least 4 months duration.

Our results suggest that in those with chronic pain after SCI, the majority develop pain within the first year, particularly in the first 6 months, but new onset of pain can occur several years after injury. Pain tends to persist, and only a small proportion resolve spontaneously. These findings have been noted by others. ${ }^{5,7,8,12,15}$ Individual SCI patients often experience more than one type of pain in terms of nature, site and severity. In line with previous reports, ${ }^{4,7,14}$ we found that in $76 \%$ of patients the pain was below the level of their SCI.

We have included all types of pain in our results and have showed that complete spinal injury is more likely to result in pain than incomplete injury. This is in contrast to previous authors who have included all types of pain in their analysis, and noted no association with, ${ }^{7,8,15}$ or an increased likelihood of pain in those with incomplete injuries. ${ }^{5,13}$ Siddall $^{15}$ found no association between the presence of pain overall and completeness of injury, but did find that neuropathic pain with allodynia was more common in people with incomplete spinal cord lesions.

We have shown that simple analgesics, weak opiates, antidepressants, anticonvulsants and TENS are used widely in the treatment of pain after SCI. The fact that $43 \%$ of patients would like further treatment for pain, suggests that current treatments are not always effective. Alternative therapies are less frequently used but may benefit certain patients. Interestingly, a handful of patients described significant pain relief from the use of cannabis. The cannabinoid nabilone is currently undergoing an assessment of its efficacy in the treatment of pain following SCI. ${ }^{16}$ Most of the published work on treatment relates to neuropathic pain. Many are case reports, and studies are often poorly controlled, retrospective, and not repeated to confirm efficacy.

We have demonstrated the major impact that pain has on the quality of life of patients after SCI, often constituting their worst problem, and being a major cause of unemployment and depression. The social and psychological consequences of chronic pain in SCI patients have been well documented..$^{2,4,5,7,12}$ In a study by Nepomuceno, ${ }^{5} 38 \%$ of patients with low thoracic or lumbar SCI, were prepared to trade the recovery from paralysis or sexual and visceral dysfunction, for pain relief.

Our study is limited in being a postal survey, but we felt that this gave a better representation of the general SCI population than would be attained by targeting those attending outpatient clinics.

This study confirms that pain is a major problem in SCI patients which is not currently being adequately addressed. A multidisciplinary approach with thorough assessment is essential if we are to improve management. This includes the involvement of pain specialists which will have resource implications. In addition, a definitive classification system for pain after SCI will aid communication of information on the subject. An International Association for the Study of Pain (IASP) working group has been created to address this issue. Finally, there is a need for 
prospective studies of interventions aimed to reduce the prevalence and severity of pain in these patients.

\section{References}

1 Stover SL, Fine PR. The epidemiology and economics of spinal cord injury. Paraplegia 1987; 25: 225-228.

2 Spinal cord injury statistics. National Spinal Cord Injury Statistical Centre, University of Birmingham, Alabama. USA.

3 Ravenscroft AJ, Ahmed YS, Burnside IG. Chronic pain after spinal cord injury. A survey of practice in UK spinal injury Units. Spinal Cord 1999; 37: 25-28.

4 Rose M, Robinson JE, Ells P, Cole JD. Pain following spinal cord injury: results from a postal survey. Pain 1988; 34: 101-102.

5 Nepomuceno $\mathrm{C}$ et al. Pain in patients with spinal cord injury. Arch Phys Med Rehab 1979; 60: 605-609.

6 Davis L, Martin J. Studies upon Spinal Cord Injuries. The nature and treatment of pain. J Neurosurgery 1947; 4: 483-491.

7 Stormer $\mathrm{S}$ et al.Chronic pain/dyaesthesia in spinal cord injury patients: results of a multicentre study. Spinal Cord 1997; 35: $446-455$.

8 Richards JS et al. Physcosocial aspects of chronic pain in spinal cord injury. Pain 1980; 8: 355-366.
9 Britell CW, Umlauf R, Loehr J, DeLisa JA. Problem survey in a SCI outpatient clinic population: a case of multifaceted, ongoing care. Arch Phys Med Rehab 1986; 67: 654.

10 Fenollosa $\mathrm{P}$ et al.Chronic pain in the spinal cord injured. Statistical approach and pharmacological treatment. Paraplegia 1993; 31: $722-729$.

11 Castellano V. Problems of analgesic therapy in traumatic paraplegia. Proc Veterans Adm Spinal Cord Inj Conf 1971; 18: $189-193$.

12 Anke AJ, Stenehjem AE, Stanghelle JK. Pain and life quality within 2 years of spinal cord injury. Paraplegia 1995; 33: $555-$ 559.

13 Demirel G, Yllmaz H, Gencosmanoglu B, Kesiktas N. Pain following spinal cord injury. Spinal Cord 1998; 36: 25-28.

14 Kennedy P, Frankel H, Gardner B, Nuseibeh I. Factors associated with acute and chronic pain following traumatic spinal cord injuries. Spinal Cord 1997; 35: 814-817.

15 Siddall PJ, Taylor DA, McClelland JM, Rutkowski SB et al. Pain report and the relationship of pain to physical factors in the first 6 months following spinal cord injury. Pain 1999; 81: 187-197.

16 Efficacy of nabilone on the treatment of pain following spinal cord injury. National research register project: No 209027449. 